\title{
Carrier-Assisted Phase Retrieval
}

\author{
Qi Wu, Yixiao Zhu, and Weisheng $\mathrm{Hu}$
}

\begin{abstract}
Phase-retrieval (PR) schemes based on the modified Gerchberg-Saxton (GS) algorithm capture the full-field employing a dispersive element and intensity-only measurements to eliminate the use of a local oscillator. In this work, we propose two carrier-assisted PR schemes, namely central carrier-assisted PR (CCA-PR) and edge carrier-assisted PR (ECA-PR), to improve the comprehensive performance of $P R$ receiver in terms of convergence speed, redundancy, and computational complexity. The proposed CCA-PR recovers the electrical field employing a reference carrier at $0 \mathrm{GHz}$ with several iterations between two projection planes. It avoids pilot symbols and digital backpropagation to the transmitter and offers a flexible electrical bandwidth requirement compared with conventional PR schemes. To lower the carrier-to-signal power ratio (CSPR) requirement and enable faster convergence for the carrier-assisted PR schemes, the ECA-PR is proposed to obtain the initial phase for the GS algorithm. We numerically characterize the performance of the two schemes and experimentally demonstrate them for 30 GBaud 16-quadrature amplitude modulation (16-QAM) transmission over $80 \mathrm{~km}$ single-mode fiber with a bit error rate (BER) below the threshold of $7 \%$ hard-decision forward error correction (HD-FEC). Several critical parameters are analyzed, including the applied dispersion value, CSPR, and electrical bandwidth. Moreover, we compare the hardware complexity and optical signal-to-noise ratio (OSNR) sensitivity of proposed PR schemes with mainstream field recovery schemes.
\end{abstract}

Index Terms-Direct detection, Phase retrieval, Gerchberg-Saxton algorithm, CSPR.

\section{INTRODUCTION}

$\mathrm{C}$ OHERENT detection has been a promising candidate for next-generation short-reach optical communications since it enables full-field recovery of dual-polarization in-phase and quadrature (IQ) modulated signals. Compared with intensity modulation and direct detection (IM-DD) schemes, the full electrical field is linearly mapped to the electrical domain by mixing the received optical field with a local oscillator (LO). Optical field impairments such as chromatic dispersion (CD) and polarization mode dispersion (PMD) can be compensated by digital signal processing (DSP) [1]. To eliminate the use of LO, some self-coherent detection schemes have been proposed to lower the cost of transceivers, including Kramers-Kronig

Manuscript received $\mathrm{xx}, \mathrm{xx}$; revised $\mathrm{xx}, \mathrm{xx}$; accepted $\mathrm{xx}, \mathrm{xx}$. Date of publication $\mathrm{xx}, \mathrm{xx}$; date of current version $\mathrm{xx}, \mathrm{xx}$. This work was supported by National Natural Science Foundation of China under Grant 62001287 and by National Key R\&D Program of China under Grant 2018YFB1800904.

Qi Wu, Yixiao Zhu, and Weisheng Hu are with the State Key Laboratory of Advanced Optical Communication Systems and Networks, Department of Electronic Engineering, Shanghai Jiao Tong University, Shanghai, 200240, China (e-mail: yixiaozhu@sjtu.edu.cn,wshu@sjtu.edu.cn). receiver (KKR) [2], Stokes-vector receiver (SVR) [3], carrier-assisted differential detection (CADD) [4], and asymmetric self-coherent detection (ASCD) [5]. At least three photodiodes (PDs) and an optical hybrid are needed in the SVR or CADD, leading to a complex hardware structure. The KKR removes signal-to-signal beating interference (SSBI) via KK relation but requires a strong carrier to meet the minimum phase condition. In other words, the carrier-to-signal ratio (CSPR) should be larger than $6 \mathrm{~dB}$ [2]. As for the ASCD, the required CSPR is about $10 \mathrm{~dB}$ for SSBI reconstruction and cancellation. However, the high CSPR requirement limits the number of wavelength division multiplexing (WDM) channels due to the limited power of Erbium-doped fiber amplifiers (EDFAs). Therefore, field recovery (FR) schemes with low CSPR and cost are preferred for larger potential capacity.

Recently, carrier-less phase-retrieval (PR) receivers based on the modified Gerchberg-Saxton (GS) algorithm [6] have been demonstrated using intensity-only measurements [7-12]. The optical full-field can be recovered employing 2 PDs and a dispersive element by searching for optimum phases that satisfy the relation between the projections. However, carrier-less detection leads to thousands of iterations and the use of pilot symbols. In [6], the PR receiver with phase reset (PR-PR) eliminates the constant phase ambiguity across the thousands of iterations using $20 \%$ pilots. The hardware cost of transceivers will be increased, although the performance of PR receivers could be enhanced by increasing the projection planes, including dispersed planes [11] or undispersed planes [12]. In [11], the PR receiver using parallel alternative projections (PAP-PR) reduces the number of iterations (NOI) to about 200 by increasing the dispersed planes. However, every additional tributary will contribute to the hardware cost of at least one PD, dispersive element, and analog-to-digital converter (ADC). Compared with PAP-PR and PR-PR, the innovative space-time diversity $\mathrm{PR}$ receiver (STD-PR) employing optical delay lines and one $3 \times 3$ coupler to mix 3 undispersed planes significantly improves the performance of PR receivers and narrows the optical signal-to-noise ratio (OSNR) sensitivity gap to about 2 $\mathrm{dB}$ compared with coherent detection [12]. Nevertheless, the additional couplers, optical delay lines, PDs, and ADCs considerably increase the cost of direct detection, making it challenging to be applied in cost-sensitive scenarios. In addition, dozens of iterations and 5\% 20\% pilot symbols are still necessary for the STD-PR receiver. The PR schemes using pilot symbols including PR-PR, PAP-PR, and STD-PR need digital backpropagation (DBP) and phase update at the transmitter, leading to high redundancy and computational complexity. We attribute the phase ambiguity to the rapid 
changes in the phase of samples, which can be eliminated using a reference carrier.

In this paper, we extend our previous work in [13] and propose two carrier-assisted phase-retrieval schemes, namely central carrier-assisted PR (CCA-PR) and edge carrier-assisted PR (ECA-PR) [13], to significantly improve the comprehensive performance of PR. The key idea of the CCA-PR is to use a central carrier at $0 \mathrm{GHz}$ to reduce the phase ambiguity in the GS algorithm. The central carrier enables CCA-PR to realize fast convergence and find the optimal solution through iteration, and the NOI can be reduced significantly. In addition, the challenge faced by all the phase retrieval receivers, including PR-PR, STD-PR, and PAR-PR, is the doubled electrical bandwidth of $P R$ receivers due to the square-law detection of the carrier-less signal. With the help of a central carrier, the required bandwidth can be flexibly adjusted according to the CSPR.

On the other hand, to further reduce the CSPR for large-scale WDM scenarios, we propose an edge carrier-assisted PR scheme (ECA-PR). The key idea of the ECA-PR is to use a weak carrier to obtain an initial phase to substitute the random phase in the conventional PR-PR because it is crucial to start gradient descent or iteration from a good initial point in the convex optimization theory. These two PR schemes show extremely 1) fast convergence speed and a dramatic reduction in the NOI for field recovery; 2) low computational complexity (no DBP to the transmitter) compared with PR-PR, PAR-PR, STD-PR; 3) simple hard-ware structure (2 PDs and a dispersive element); and 4) low redundancy (no pilot symbols). We then experimentally validate the principle and feasibility of the 2 PR schemes in a 30 GBaud 16-quadrature amplitude modulation (16-QAM) direct detection system. The theoretical performance and hardware cost of some field recovery schemes are compared.

This paper is organized as follows. In section II, we introduce the principle of two carrier-assisted PR schemes and analyze the conditions to demonstrate PR. In Section III, we numerically show the singularity in the CCA-PR scheme, and the influence factors of the singularity are also presented. Simulations are performed to verify the principle and feasibility of these two schemes and compare them with conventional PR receivers. The transmission experiments of $120 \mathrm{Gbit} / \mathrm{s}$ over 80$\mathrm{km}$ are conducted in Section IV to investigate the impact of CSPR, guard band, and electrical noise on carrier-assisted PR schemes. We compare and analyze the key parameters of some mainstream FR schemes, including hardware complexity, CSPR, and OSNR sensitivity in Section V. Finally, Section VI makes a summary of this paper.

\section{PRINCIPLE}

The structures of CCA-PR and ECA-PR are shown in Fig. 1. It consists of 2 single-ended PDs and a dispersive element, which is the same as PR-PR. $a(t)$ and $b(t)$ are the intensity measurements on the undispersed plane and dispersed plane, respectively. $D$ is the dispersive element. For the CCA-PR, the difference is that the carrier is used to reduce the variance of phases at the transmitter and thus we modify the corresponding

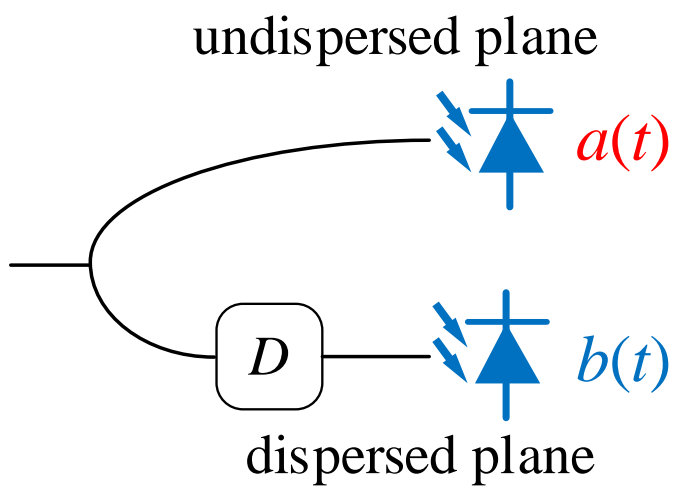

Fig. 1. Receiver structures of the CCA-PR and ECA-PR schemes.

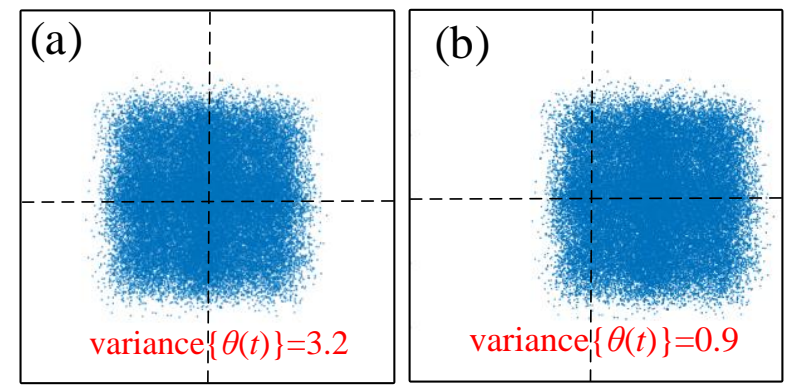

Fig. 2. Constellations of QPSK signal $x(t)$ without and with a carrier at -1 dB CSPR.

GS algorithm at the receiver DSP for phase retrieval. For the ECA-PR, the carrier is aimed to capture an initial phase to enable faster convergence than CCA-PR and we also modify the GS algorithm with phase initialization at the receiver DSP for PR at low CSPR.

\section{A. Principle of the CCA-PR}

The constellations of transmitted signal $x(t)$ shaped by a raised root filter (RRC) with 0.01 roll-off factor in the IQ-plane without and with a carrier at $0 \mathrm{GHz}$ are depicted in Fig. 2(a)-(b). The samples without a carrier are distributed around the origin. The rapidly changing phase makes it difficult for the GS algorithm to converge and thus it requires the pilot symbols and thousands of iterations to eliminate phase ambiguity [7]. We propose to eliminate the phase ambiguity using a carrier at 0$\mathrm{GHz}$ and thus PR can be realized without the pilot symbol. We use $\theta(t)$ to represent the phase of the samples, given as:

$$
\theta(t)=\angle\{x(t)\}
$$

$x(t)$ is the transmitted complex signal and $\angle$ is the angle function. The unit of $\theta(t)$ is radian, ranging from $-\pi$ to $\pi$. The speed of phase change can be described using the variance of $\theta(t)$. The variance of $\theta(t)$ is 3.2 for $x(t)$ at 2 samples-per-symbol (SPS) without a carrier. Assisted with a carrier at $0 \mathrm{GHz}$, the speed of phase change can be reduced dramatically, which is shown in Fig. 2(b). The CSPR of quadrature phase shift keying (QPSK) signal is set as $-1 \mathrm{~dB}$. The variance of $\theta(t)$ could be reduced from 3.2 to 0.9 . The carrier at $0 \mathrm{GHz}$ enables the GS algorithm to retrieve the optical field and realize fast convergence.

Then we analyze the requirement for CCA-PR to improve 


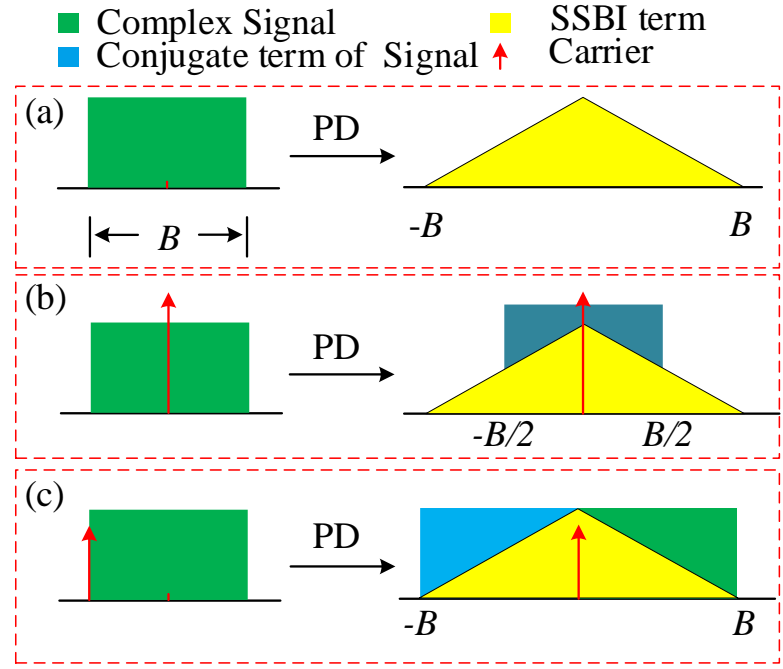

Fig. 3. Schematic diagram of square-law detection in the (a) conventional PR schemes; (b) CCA-PR scheme and (c) ECA-PR scheme.

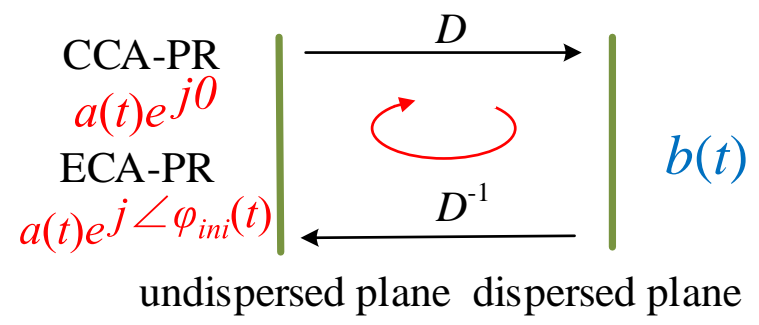

Fig. 4. Schematics of the modified GS algorithm for CCA-PR and ECA-PR schemes.

electrical spectral efficiency (ESE). As shown in Fig. 3(a), the bandwidth of SSBI generated by photoelectric conversion is twice the signal spectrum. Assuming that the bandwidth of the signal is $B$, the requirement for the electrical bandwidth at the transmitter is $B / 2$ while it is $B$ for the conventional phase retrieval receiver to demonstrate field recovery. Compared with the intradyne coherent detection scheme, the ESE is almost halved. In order to improve the ESE of PR schemes, we enhance the carrier power to concentrate the power of photocurrent $I(t)$ in the low-frequency region. The principle could be explained as:

$$
I(t)=|C+S(t)|^{2}=C C^{*}+C^{*} S(t)+C S(t)^{*}+|S(t)|^{2},
$$

where $C$ is the carrier located at the $0 \mathrm{GHz}$ and $S(t)$ is the optical complex signal. $*$ is the conjugate symbol. We can see the energy of $I(t)$ consists of DC component $\left(C C^{*}\right)$, the signal term $\left(C^{*} S(t)\right)$, the conjugate term of signal $\left(C S(t)^{*}\right)$, and SSBI term $\left(|S(t)|^{2}\right)$. The schematic diagrams of square-law detection in the CCA-PR scheme are depicted in Fig. 3(b).

To mitigate the impact of the SSBI term outside $B / 2$ on the PR algorithm, we concentrate the power in the range $-B / 2$ to $B / 2$ employing a strong carrier located in $0 \mathrm{GHz}$. The power of the SSBI term outside $B / 2$ is neglectable compared with linear terms if the CSPR is sufficiently large and thus the bandwidth of $B / 2$ is sufficient for the PR receiver to receive the dominant term within $B / 2$. Assisted with a carrier, the phase can be
TABLE I

MODIFIED GS ALGORITHM FOR CCA-PR

\begin{tabular}{lll}
\hline \hline \multicolumn{2}{l}{ function CCA-PR $\left(a(t), b(t), h_{D}(t)\right)$} & \\
1 & $r(t)=1$ & Initialize the phases \\
2 & $a(t)=a(t) / \max (a(t))$ & Amplitude normalization \\
3 & $b(t)=b(t) / \max (b(t))$ & Amplitude normalization \\
4 & For i in $\mathrm{M}$ do & M iterations between 2 planes \\
5 & $r(t)=a(t) \exp (j \angle r(t))$ & Intensity constraint \\
6 & $d(t)=r(t) \otimes h_{D}(t)$ & Apply projection \\
7 & $d(t)=b(t) \exp (j \angle d(t))$ & Intensity constraint \\
8 & $d(t)=d(t) \otimes h_{B W}(t)$ & Spectral constraint \\
9 & $r(t)=d(t) \otimes h^{-1}(t)$ & Remove projection \\
10 & $\operatorname{Return} r(t)$ & End iteration \\
\hline
\end{tabular}

retrieved with halved electrical bandwidth.

Fig. 4 illustrates the CCA-PR scheme, which is operated between the undispersed plane and the dispersed planes to retrieve phases. The DBP to transmitter and phase update by the pilot symbols are no longer needed for this scheme.

The modified GS algorithm for the CCA-PR is shown in Table I. The max is the maximum value function. $h_{D}(t)$ is the transfer function for chromatic dispersion from the dispersive element inside the PR receiver. $h_{B W}(\mathrm{t})$ is a spectral filter to match the signal bandwidth. Note that $h_{B W}(\mathrm{t})$ is a band-pass filter with 2 windows in the CCA-PR due to the singularity at 0 $\mathrm{GHz}$, which will be explained in the next section. $\otimes$ is the convolution symbol. $r(t)$ is the recovered signal.

The initial phase is set as zeros. $a(t)$ and $b(t)$ should be normalized first. After applying projection, a spectral brick-wall filter is used to constrain the spectrum. Then the projection is removed, and the intensity constraint is applied. With the help of the carrier, the phase can be retrieved with several iterations by the modified GS algorithm for CCA-PR.

\section{B. Principle of the ECA-PR}

A CSPR of more than $3 \mathrm{~dB}$ is needed to relax the bandwidth requirement in the CCA-PR, which leads to a substantial OSNR sensitivity penalty. In addition, a high CSPR limits the number of WDM channels. Then we further propose the ECA-PR to lower CSPR for the carrier-assisted PR schemes. The weak carrier is inserted at the edge of the signal spectrum shown in Fig. 3(c), and thus the variance of $\theta(t)$ will not be reduced. To obtain an initial phase $\varphi_{\text {ini }}(t)$ for the GS algorithm and escape from the local minima, a weak carrier at $B / 2$ is required. The optical field of the undispersed plane could be denoted as $W_{c} e^{-j \pi B t}+S(t)$, where $W_{C}, S(t)$, and $B$ are the weak carrier, optical complex signal, and signal bandwidth, respectively. The accurate phase of the optical field is $\angle\left\{W_{c} e^{-j \pi B t}+S(t)\right\}$. After the $\mathrm{PD}$, the photocurrent detected on the undispersed plane could be written as:

$$
|a(t)|^{2}=\left|W_{C} e^{-j \pi B t}+S(t)\right|^{2}=\left|W_{C}\right|^{2}+W_{C}{ }^{*} e^{j \pi B t} S(t)+W_{C} e^{-j \pi B t} S(t)^{*}+|S(t)|^{2} .
$$

Assisted with the weak carrier at $B / 2$, we can obtain an initial phase distorted by signal-to-signal beat noise, given as:

$\varphi_{i n i}(t)=\angle\left\{R\left[|a(t)|^{2} / W_{C}^{*}\right] e^{-j \pi B t}\right\}=\angle\left\{W_{C} e^{-j \pi B t}+S(t)+R\left[|S(t)|^{2} / W_{C}^{*}\right] e^{-j \pi B t}\right\}$, 

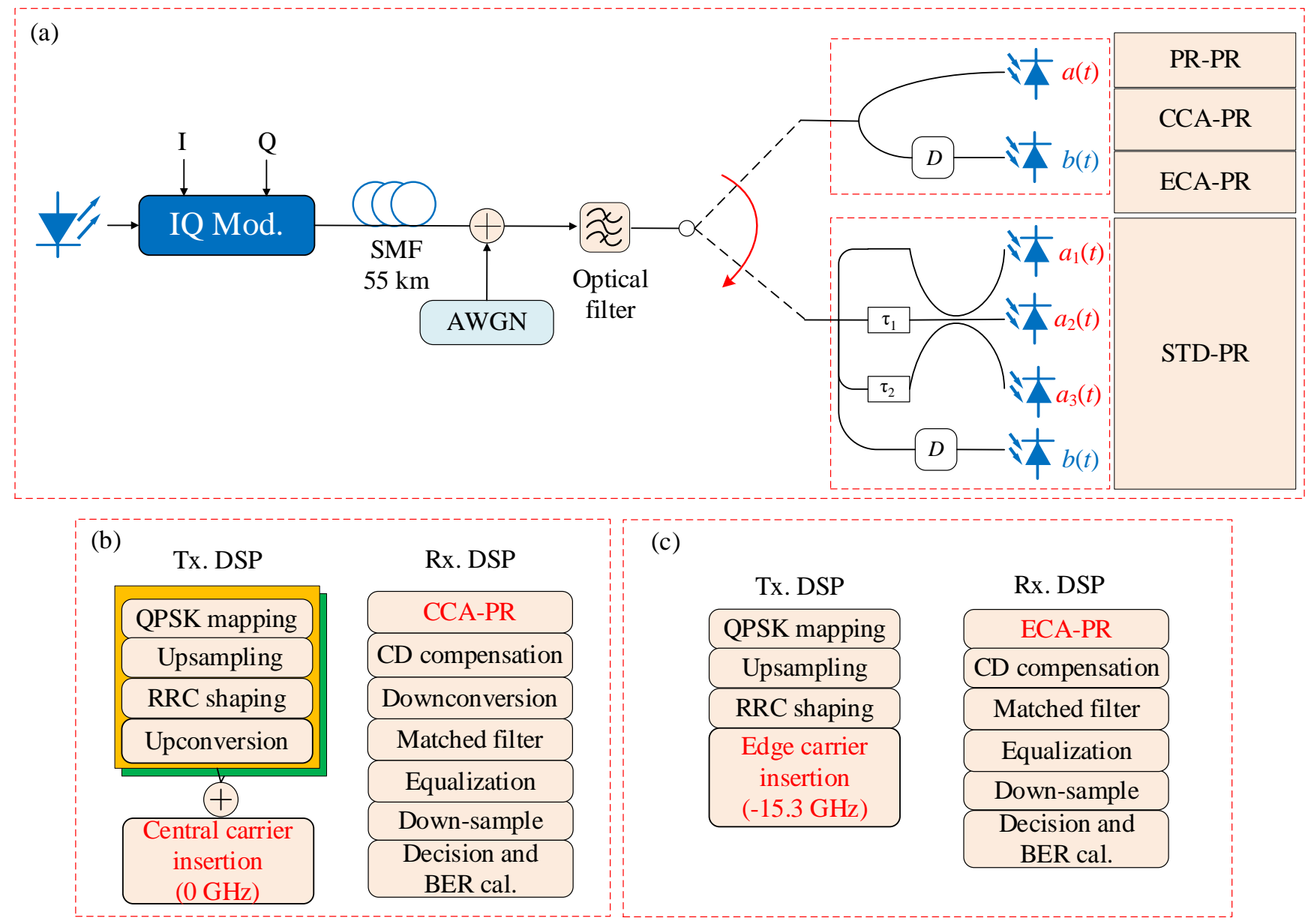

Fig. 5. (a) Simulation setup of the PR schemes; (b) DSP stacks for CCA-PR; (c) DSP stacks for ECA-PR; Tx.: transmitter; Rx.: receiver.

where $R[$.$] denotes a sideband filter aimed to remove$ conjugation term $W_{C} e^{-j \pi B t} S(t)^{*}$. The schematic diagrams of square-law detection in the ECA-PR scheme are depicted in Fig. 3 (c). The initial phase is necessary for the ECA-PR, although it is distorted severely.

Table. II illustrates the modified GS algorithm with phase initialization (MGS-PI) for the proposed ECA-PR. The schematic diagram of the algorithm is also shown in Fig. 4. In this algorithm, $\varphi_{\text {ini }}(t)$ is the obtained initial phase distorted by the SSBI. The procedure of MGS-PI also consists of amplitude normalization, intensity constraint, projection, spectral constraint, projection removal. The diffidence is that we take the $\varphi_{i n i}(t)$ as the initial phase and then conduct the MGS-PI algorithm just between 2 projection planes. After several iterations, the optical field could be retrieved.

Compared with the algorithm used in conventional PR schemes using pilot symbols [7-12], these 2 PR schemes eliminate the use of pilot symbols, leading to reduced redundancy. The NOI can be reduced to several times, and thus the computational complexity is also reduced. It is unnecessary for the CCA-PR and ECA-PR to backpropagate the reconstructed optical field to the transmitter for phase correction. The imperfections of the transmitter and fiber link have little impact on them.
TABLE II

MODIFIED GS ALGORITHM WITH PHASE INITIALIZATION FOR CCA-PR

\begin{tabular}{lll}
\hline \multicolumn{2}{l}{ Function ECA-PR $\left(a(t), b(t), \varphi_{\text {ini }}(t)\right.}$, & $\left.h_{D}(t)\right)$ \\
1 & $r(t)=\exp \left(j \varphi_{\text {inin }}(t)\right)$ & Initialize the phases \\
2 & $a(t)=a(t) / \max (a(t))$ & Amplitude normalization \\
3 & $b(t)=b(t) / \max (b(t))$ & Amplitude normalization \\
4 & For i in M do & M iterations between 2 planes \\
5 & $r(t)=a(t) \exp (j \angle r(t))$ & Intensity constraint \\
6 & $d(t)=r(t) \otimes h_{D}(t)$ & Apply projection \\
7 & $d(t)=b(t) \exp (j \angle d(t))$ & Intensity constraint \\
8 & $d(t)=d(t) \otimes h_{B W}(t)$ & Spectral constraint \\
9 & $r(t)=d(t) \otimes h^{-1}(t)$ & Remove projection \\
10 & $\operatorname{Return} r(t)$ & End iteration \\
\hline
\end{tabular}

\section{SimULATIONS}

In this section, we conduct simulations to numerically characterize the performance of proposed CCA-PR and ECA-PR. For the CCA-PR, the singularity at $0 \mathrm{GHz}$, NOI and CSPR requirement are analyzed. For the ECA-PR, the necessity of the initial phase $\varphi_{i n i}(t)$, NOI and the applied dispersion value $(\mathrm{ADV})$ inside the $\mathrm{PR}$ receiver are also presented.

\section{A. Fast convergence and flexibility with CCA-PR}

We find an interesting phenomenon in the CCA-PR receiver 

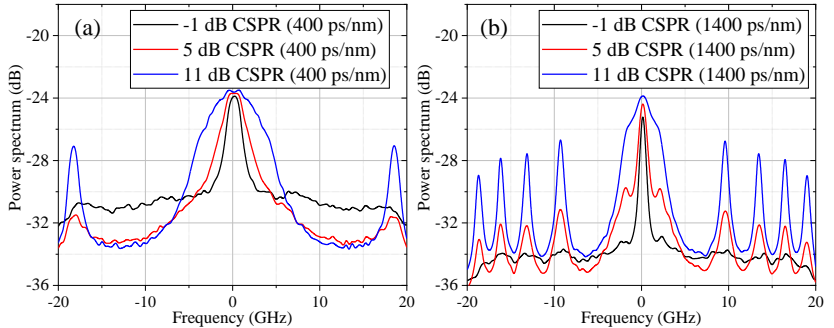

Fig. 6. Power spectral density of residual error at different CSPRs with ADV of (a) $400 \mathrm{ps} / \mathrm{nm}$ and (b) $1400 \mathrm{ps} / \mathrm{nm}$.

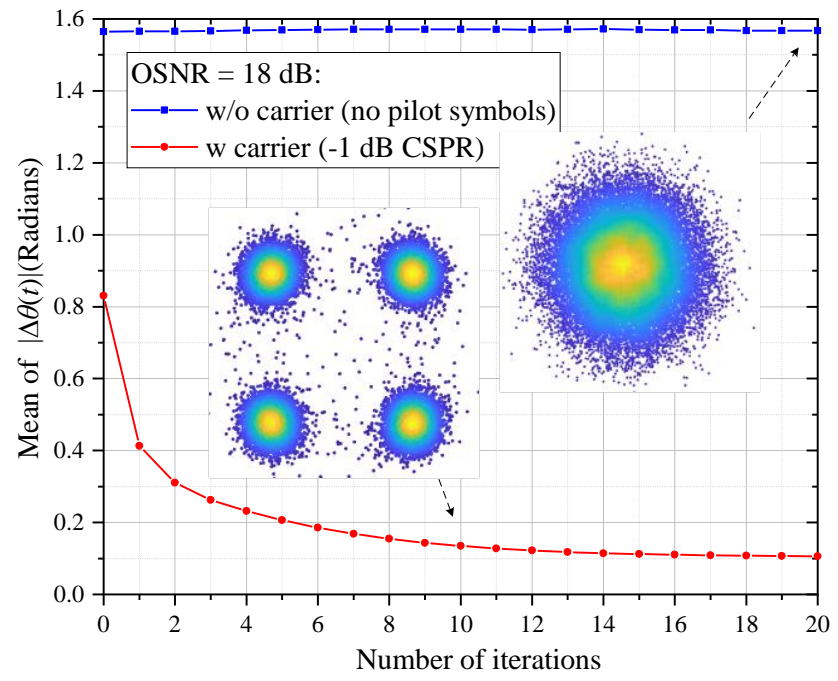

Fig. 7. Mean of $|\Delta \theta(t)|$ versus the number of iterations for CCR scheme with and without a carrier under $18 \mathrm{~dB}$ OSNR.

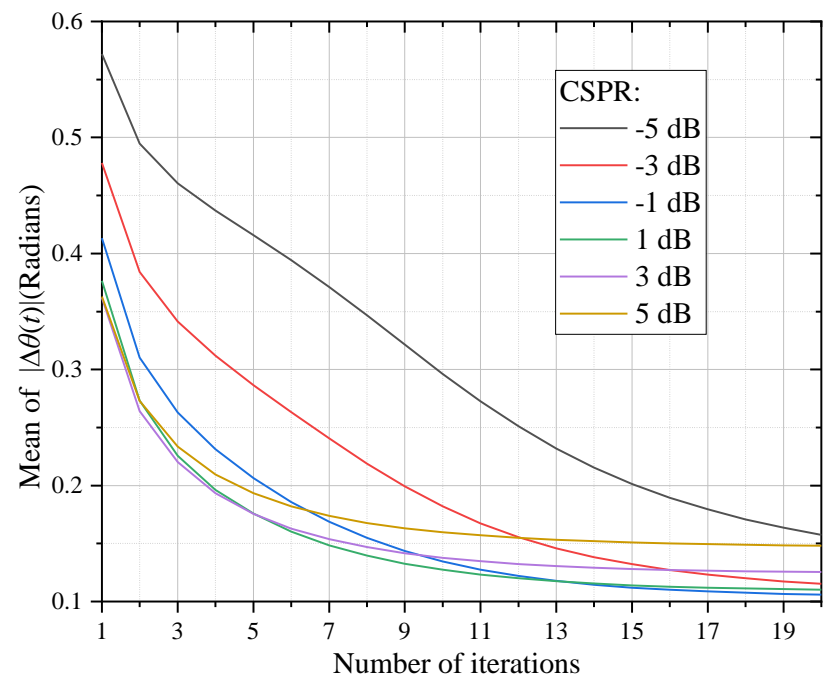

Fig. 8. Mean of $|\Delta \theta(t)|$ versus the number of iterations for CCA-PR scheme at different CSPRs.

that there is a singularity at $0 \mathrm{GHz}$. This phenomenon also appears in other complex double-sideband self-coherent detection schemes such as CADD and ASCD. To illustrate this phenomenon, we conduct simulations to investigate the parameters related to the singularity. We perform a simulation with a twin-single-sideband (SSB) 40 GBaud QPSK signal at the back-to-back (BTB) case. The simulation setup and DSP stack for CCA-PR are shown in Fig. 5(a)-(b), respectively.
To quantitatively describe the singularity, we define the power spectral density of residual error between the recovered $r(t)$ and the transmitted signal $x(t)$ as:

$$
F_{e r r}(w)=|F F T\{x(t)-r(t)\}|,
$$

where FFT is the Fourier transform. The residual frequency error $F_{\text {err }}(w)$ in the frequency range of $-20 \mathrm{GHz}$ to $20 \mathrm{GHz}$ at different CSPRs with ADV of $400 \mathrm{ps} / \mathrm{nm}$ and $1400 \mathrm{ps} / \mathrm{nm}$ are shown in Fig. 6(a)-(b), respectively.

The singularities except for the one at $0 \mathrm{GHz}$ are caused by using the modified GS algorithm based on the dispersive element to retrieve the phase of a carrier-assisted double-sideband signal, which is analyzed in [14]. We can see the impact of singularities becomes severe as the CSPR increases. In the $1400 \mathrm{ps} / \mathrm{nm}$ case, there are eight singularities in the intermediate frequency (IF) range. The results indicate that the slope and the location of singularities depend on the CSPR and the applied dispersion value, respectively. The higher CSPR leads to the larger singularities, and the larger applied dispersion moves the singularities towards $0 \mathrm{GHz}$.

Fortunately, the system performance penalty mainly comes from the singularity at $0 \mathrm{GHz}$, which be prevented by a guard band. To match the experiment using a dispersive element, we mitigate the impact of the singularity at $0 \mathrm{GHz}$ using a $3 \mathrm{GHz}$ guard band in the following simulation. We then conduct a twin-SSB 30 GBaud QPSK simulation to show the convergence of the CCA-PR. The single-mode fiber (SMF) link is $55 \mathrm{~km}$, and the ADV inside the CCA-PR receiver is set as 650 $\mathrm{ps} / \mathrm{nm}$. According to [7, 11], some definitions are as follows. $A_{\text {err }}(t)$ is the sample-wise amplitude error used to reset the phases for PR-PR scheme, which is calculated by the amplitude difference between the recovered $r(t)$ and the measured $a(t)$.

$$
A_{e r r}(t)=\left.|a(t)-| r(t)\right|^{2}
$$

The symbol-wise absolute phase error $|\Delta \theta(t)|$ is the absolute phase difference between the transmitted QAM symbol and the recovered QAM symbol, denoted as:

$$
|\Delta \theta(t)|=\left|\angle\left\{T x S(t) R x S(t)^{*}\right\}\right|,
$$

where $\operatorname{Tx} S(t)$ is the transmitted QAM symbol and $\operatorname{Rx} S(t)$ is the recovered QAM symbol.

Fig. 7 shows the mean of $|\Delta \theta(t)|$ versus the NOI without and with the carrier under $18 \mathrm{~dB}$ OSNR. We can see that the mean of $|\Delta \theta(t)|$ has no downward trend without carrier or pilot symbol while the carrier helps to reduce variance and enables fast convergence. Fig. 8 shows the mean of $|\Delta \theta(t)|$ versus the NOI at different CSPRs. It can be observed that the larger the CSPR, the faster the modified GS algorithm converges. The mean of $|\Delta \theta(t)|$ after 20 iterations increases when CSPR exceeds $1 \mathrm{~dB}$ due to the limited OSNR. As the CSPR increases, the absolute phase error induced by amplified spontaneous emission (ASE) noise increases.

We simulate the required brick-wall electrical bandwidth versus CSPR at the cost of $1 \mathrm{~dB}$ OSNR to validate the ESE could be improved by a higher CSPR. The OSNR sensitivity with $1 \mathrm{~dB}$ penalty is from $19 \mathrm{~dB}$ to $18 \mathrm{~dB}$. The results are displayed in Fig. 9. It shows the penalty from the insufficient bandwidth is alleviated by a higher CSPR. When CSPR is 


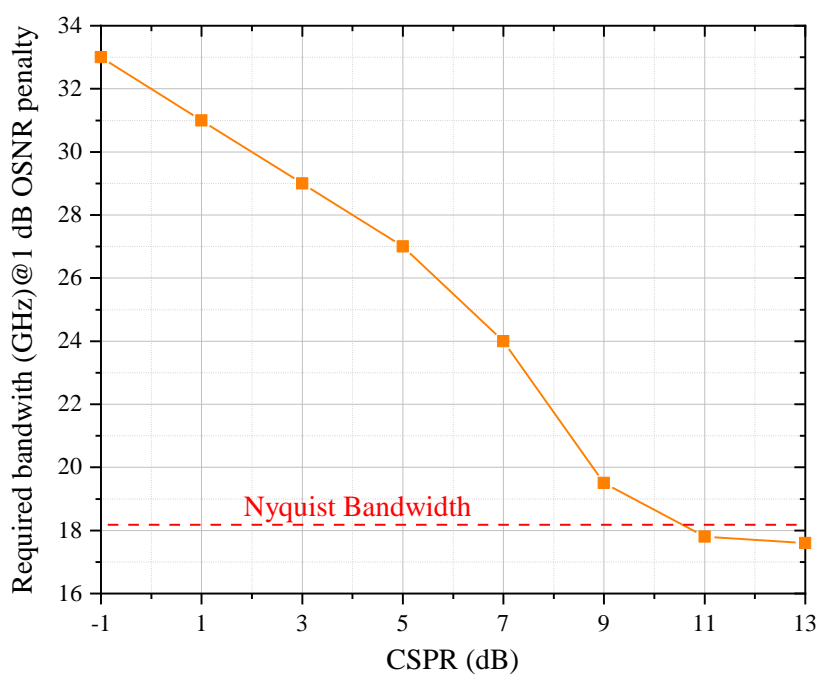

Fig. 9. Required brick-wall bandwidth versus CSPRs at $1 \mathrm{~dB}$ OSNR penalty.

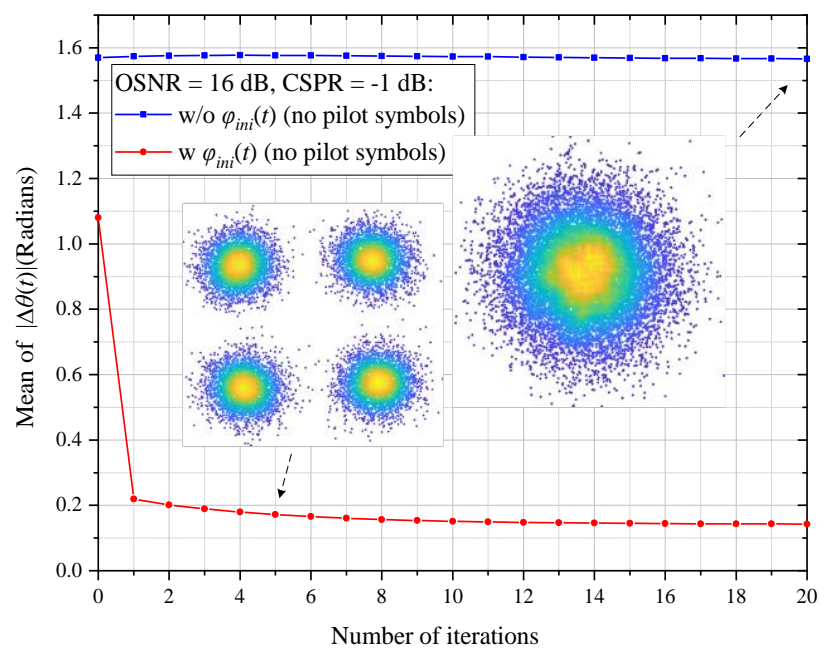

Fig. 10. Mean absolute phase error $|\Delta \theta(t)|$ versus the number of iterations for ECA-PR scheme with and without phase initialization.

beyond $11 \mathrm{~dB}$, the power of SSBI outside $18 \mathrm{GHz}$ has little impact on the system performance. Accordingly, the ESE can be improved based on enhancing the power of the carrier. The penalty caused by increasing CSPR is the OSNR sensitivity. It is a trade-off between OSNR sensitivity and ESE. The above simulations imply that the CSPR of more than $3 \mathrm{~dB}$ is required for a higher ESE of PR schemes, which lessens the number of WDM channels.

\section{B. Low CSPR with ECA-PR}

We then perform numerical simulations to validate the principle and feasibility of the proposed ECA-PR and compare it with the conventional PR-PR and STD-PR. The DSP stack for ECA-PR is shown in Fig. 5(c). We numerically simulate single-carrier 30 GBaud QPSK transmission over $55 \mathrm{~km} \mathrm{SMF}$ to match the previous work $[7,12]$. The ADV is $650 \mathrm{ps} / \mathrm{nm}$. To simplify the performance comparison, the phase reset parameter is 200 and the acceptable error level is 0.002 $\left(A_{\text {err }}(t)>0.002\right)$ for conventional PR-PR, which is an
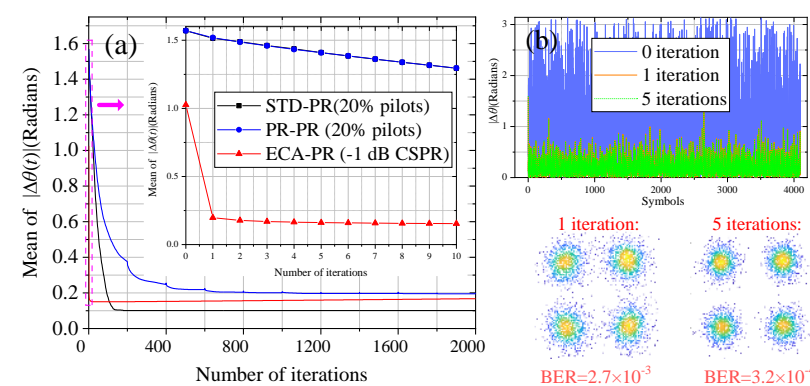

Fig. 11. (a) Mean absolute phase error $|\Delta \theta(t)|$ versus the NOI; (b) Absolute phase error of symbol sequence and constellations after 1 iteration and 5 iterations.

appropriate setting according to [7]. The number of total iterations is set at 2000 to ensure that the GS algorithm converges, and $20 \%$ periodically inserted pilot symbols are used for PR-PR and STD-PR.

First, we show the results with and without an initial phase. The mean absolute phase error $|\Delta \theta(t)|$ versus the NOI with and without $\varphi_{i n i}(t)$ under $16 \mathrm{~dB}$ OSNR are displayed in Fig. 10.

The CSPR is set as $-1 \mathrm{~dB}$. It shows that the initial phase $\varphi_{i n i}(t)$ is crucial in the ECA-PR receiver, and the PR algorithm will not work without the help of $\varphi_{\text {ini }}(t)$ since the carrier at IF could not lower variance of $\theta(t)$.

The mean absolute phase error $|\Delta \theta(t)|$ versus the NOI with 3 PR schemes under $16 \mathrm{~dB}$ OSNR at the BTB scenario are displayed in Fig. 11(a) to show the extremely fast convergence speed of ECA-PR.

The process of the first ten iterations is shown in the inset. For PR-PR/STD-PR and ECA-PR, the initial mean of $|\Delta \theta(t)|$ are 1.57 and 1.06, respectively. After 1 iteration and 5 iterations, the mean of $|\Delta \theta(t)|$ for ECA-PR is 0.19 and 0.17 , respectively. The absolute phase error of symbol sequence after 0 iteration, 1 iteration, and 5 iterations and constellations are depicted in Fig. 11(b). It shows only one iteration is needed to reach the bit error rate (BER) threshold of 7\% hard-decision forward error correction (HD-FEC) and takes 5 iterations to realize full phase retrieval. The residual absolute phase error results from the Gaussian noise.

Then, we investigate the impact of the ADV on the performance of ECA-PR and PR-PR. Fig. 12 depicts the converged BER with respect to the ADV under $16 \mathrm{~dB}$ OSNR. It shows that the required applied dispersion decreases and with the CSPR increasing from $-2 \mathrm{~dB}$ to $2 \mathrm{~dB}$. This implies that the CSPR can be further reduced if the ADV is large enough, and thus the OSNR penalty caused by the power of the carrier will be smaller.

The NOI required in the ECA-PR depends on the CSPR for a fixed ADV. Fig. 13 depicts the simulated BERs versus CSPR under different OSNRs and the NOI versus CSPR under $18 \mathrm{~dB}$ OSNR after $55 \mathrm{~km}$ transmission. With the OSNR increasing, the optimal CSPR increases to obtain better BER performance. It is a trade-off between effective OSNR and convergence error results from the GS algorithm. As the CSPR increases, the NOI 
required can be reduced because the $\varphi_{i n i}(t)$ will be closer to the true phase.

\section{TRANSMISSION EXPERIMENTS}

\section{A. Transmission Experiment for the CCA-PR}

Fig. 14 illustrates the experimental setup. In the transmitter DSP, we generate two independent 15 GBaud 16-QAM signals with a length of $2^{12}$. After up-sampling to 8 SPS and RRC filter with a roll-off factor of 0.01 , the two independent signals are up-converted to the IF to accommodate the $1 / 2 / 3 \mathrm{GHz}$ guard bands and combined into a twin-SSB signal. The I/Q components of the merged signal are generated by an arbitrary waveform generator (Keysight M8194A) of $45 \mathrm{GHz} 3-\mathrm{dB}$ bandwidth, operating at $120 \mathrm{GSa} / \mathrm{s}$ and amplified by a dual-channel electrical amplifier (OA4SMM4). Then the electrical signals are loaded into an IQ modulator of $25 \mathrm{GHz} 3-$ $\mathrm{dB}$ bandwidth to modulate the light from an external cavity laser (ECL) with a linewidth of $\sim 100 \mathrm{kHz}$. The CSPR is controlled by the bias point of the IQ modulator for the CCA-PR, while a virtual carrier is introduced in Tx-DSP, and the IQ modulator is kept biasing at the null point for the ECA-PR scheme. The optical signal is amplified to $0 \mathrm{dBm}$ by an EDFA and launched to $80 \mathrm{~km} \mathrm{SMF}$. At reception, the optical signal is firstly amplified to $18 \mathrm{dBm}$ and filtered using an optical bandpass filter (OBPF). Here, the variable optical attenuator (VOA) is used to control OSNR. Then, the optical signal is spilt using a 30:70 coupler. The tributary of $70 \%$ power is dispersed employing a dispersion compensation module (TDCMX-SM TERAXION) with a tunable dispersion value ranging from -1413 to $0 \mathrm{ps} / \mathrm{nm}$. The dispersion compensation module (DCM) is based on Fiber Bragg grating. The insertion loss of the DCM is $3.5 \mathrm{~dB}$, and the relative optical delay is about 25000 ps which is calculated in advance according to the synchronization peak with IM-DD configuration. The received optical power of both the undispersed plane and the dispersed plane is $7 \mathrm{dBm}$ approximately. The two optical signals are detected by two PDs (XPD3120R) and digitized by a real-time digital storage oscilloscope (DSO) with a $33 \mathrm{GHz}$ brick-wall electrical bandwidth (Tektronix DPO75902SX) operating at $100 \mathrm{GSa} / \mathrm{s}$ for offline DSP.

At the receiver DSP, after resampling to $120 \mathrm{GSa} / \mathrm{s}$, we perform synchronization through the correlation between the sent and received SSB synchronization frames. The two waveforms are resampled to $90 \mathrm{GSa} / \mathrm{s}$ to avoid spectral overlap, and then the modified GS algorithm for CCA-PR (Table. I) is used to realize field recovery. Note that the spectral filter $h_{B W}(t)$ in the modified GS algorithm for CCA-PR should be replaced by a dual-passband filter to match the transmitted spectrum due to the guard band. Next, the recovered signal is resampled to 2-

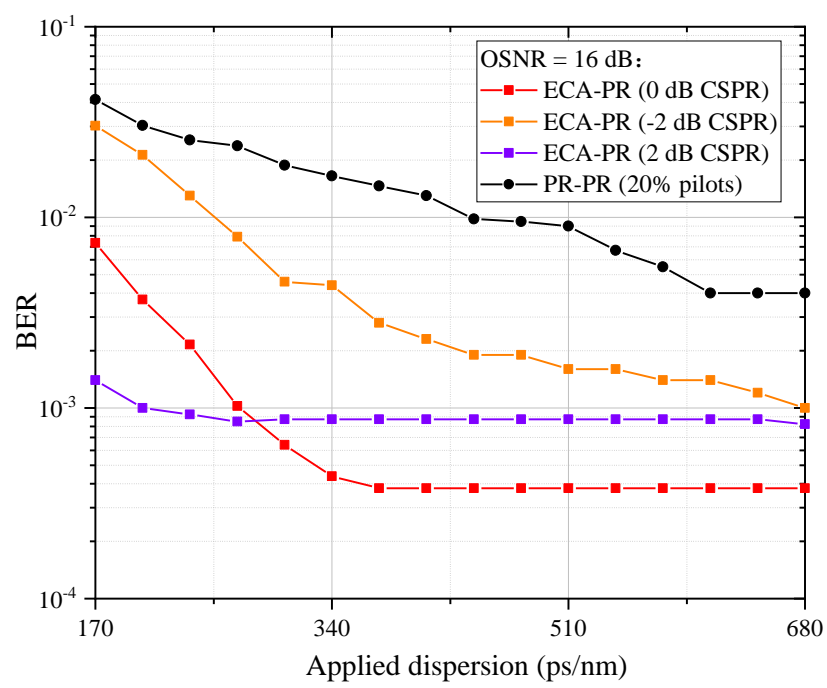

Fig. 12. Simulated BERs versus applied dispersion value at different CSPRs in the PR receiver.

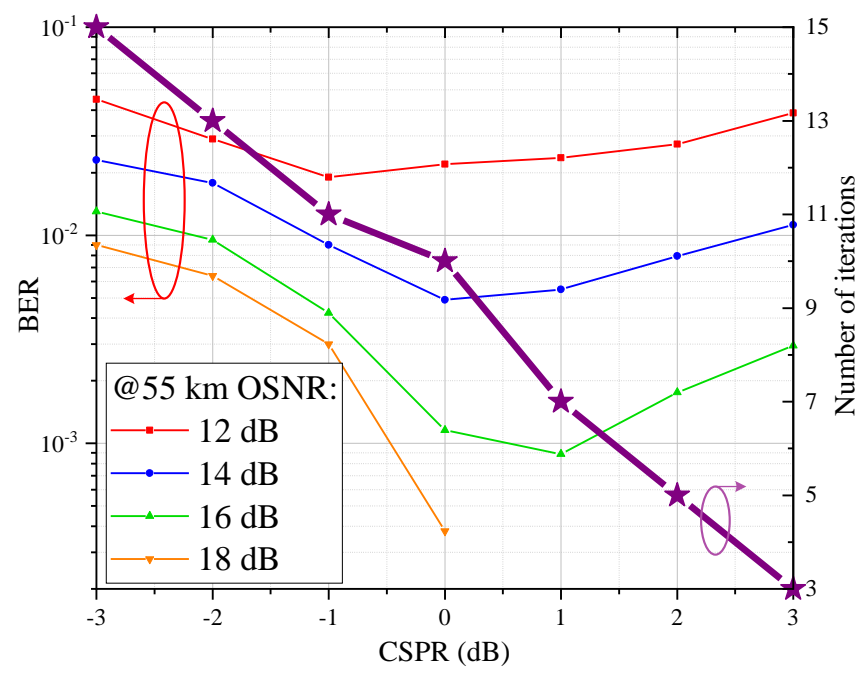

Fig. 13. Simulated BERs versus CSPR under different OSNRs and the number of iterations versus CSPR under $18 \mathrm{~dB}$ OSNR.

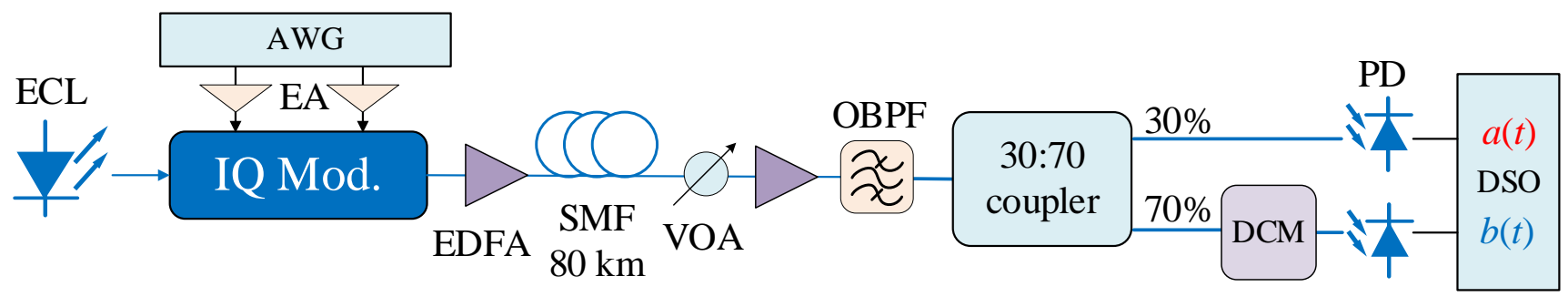

Fig. 14. Experimental setup for the CCA-PR and ECA-PR. ECL: external cavity laser; AWG: arbitrary waveform generator; IQ Mod.: IQ modulator; EA: electrical amplifier; EDFA: erbium-doped fiber amplifier; VOA: variable optical attenuator; PD: photodetector; OBPF: optical band-pass filter; DCM: dispersion compensation module; DSO: digital storage oscilloscope. 
SPS. After CD compensation, down-conversion, and matched filter, a sparse Volterra series nonlinear decision feedback equalizer (VNDFE) is used to compensate for the modulation nonlinearity and eliminate inter-symbol interference (ISI). The number of taps is $31,5,3,2$ for $1^{\text {st }},-2^{\text {nd }}-, 3^{\text {rd }}-$, and decision feedback branch, respectively. The tap coefficients are updated by the recursive least square (RLS) algorithm based on 128 training symbols. After down-sampling and decision, the BER is calculated. The DSP procedures are shown in Fig. 15, and the optical spectra of different CSPR are depicted in Fig. 16.

Assisted with the modulator carrier, the CCA-PR enables fast convergence. In the $80 \mathrm{~km}$ transmission case, the measured mean of $|\Delta \theta(t)|$ versus the NOI under different CSPRs is displayed in Fig. 17.

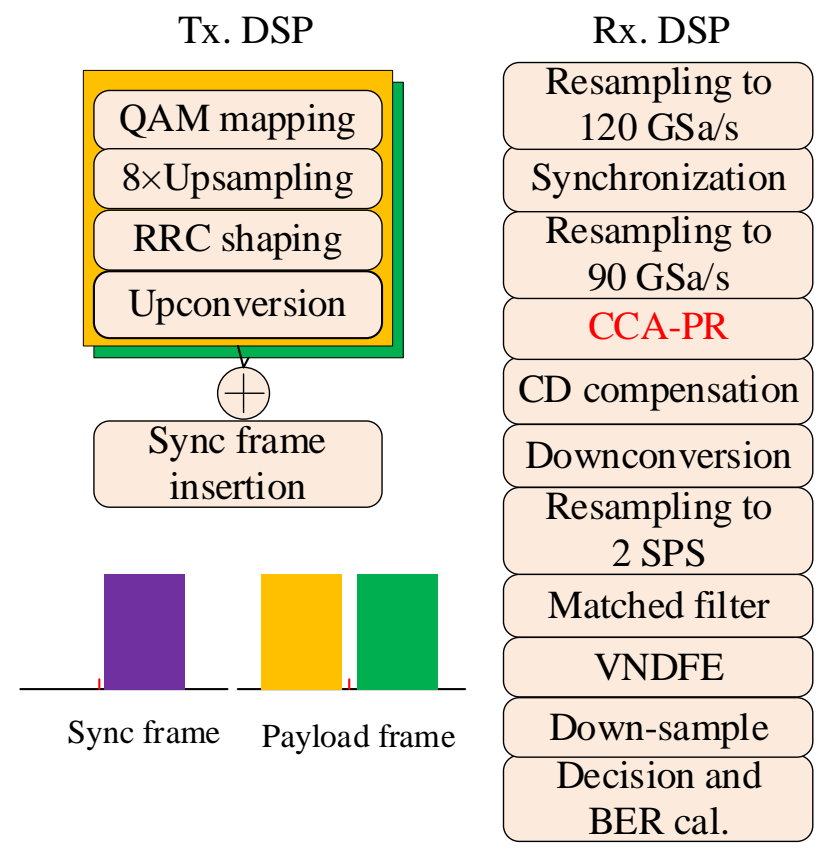

Fig. 15. DSP stacks for CCA-PR at the transmitter and receiver.

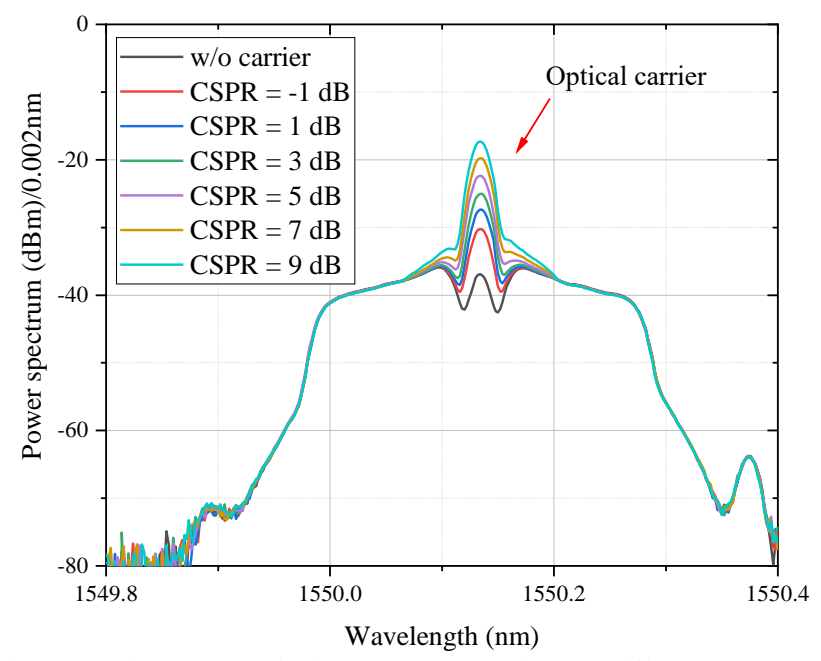

Fig. 16. Optical spectra of twin-SSB 16-QAM signal at different CSPRs.

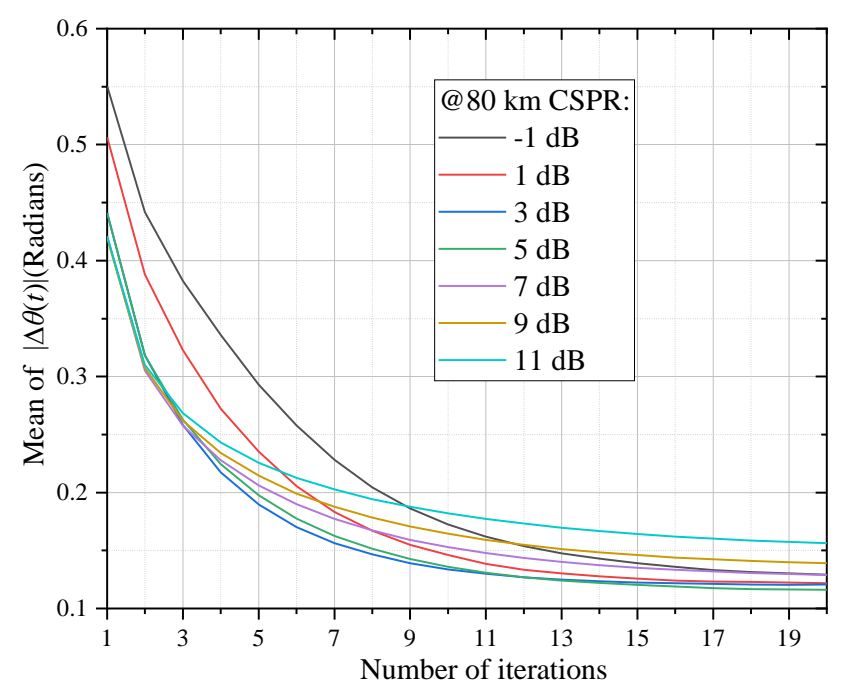

Fig. 17. Mean of $|\Delta \theta(t)|$ versus the number of iterations at different CSPRs after $80 \mathrm{~km}$ SMF transmission.

The guard band is $3 \mathrm{GHz}$ and the ADV is $-1400 \mathrm{ps} / \mathrm{nm}$. With the CSPR increasing from $-1 \mathrm{~dB}$ to $5 \mathrm{~dB}$, the convergence speed increases and the mean of $|\Delta \theta(t)|$ under 20 iterations decreases. However, a higher CSPR degrades the performance of CCA-PR, although the convergence speed increases because the OSNR penalty from the carrier power become larger and the modulation nonlinearity is also enhanced. In the following context, the NOI is set as 15 . Next, we characterize the performance impact of the guard band and CSPR. Fig. 18 shows the measured BERs as a function of the CSPR with the guard band from $1 \mathrm{GHz}$ to $3 \mathrm{GHz}$. It shows that the guard band can effectively mitigate the penalty from the singularity at 0 GHz. The optimal CSPR is about $7 \mathrm{~dB}$ for the PR receiver to realize full-field recovery.

We emulate the brick-wall electrical bandwidth in the receiver DSP to investigate the tolerance against bandwidth limitation. The BERs as a function of brick-wall bandwidth are displayed in Fig. 19. It shows that a higher CSPR helps to concentrate the signal power in the low-frequency region and thus be more tolerant to electrical bandwidth limitation at the receiver. $25 \mathrm{GHz}$ is sufficient for the CCA-PR to reach a BER below $3.8 \times 10^{-3}$ while $30 \mathrm{GHz}$ is required for the traditional PR schemes.

\section{B. Transmission Experiment for the ECA-PR}

We experimentally validate the principle of the ECA-PR in a single carrier 30 GBaud QPSK/16-QAM transmission system. The experimental setup is the same as the CCA-PR. The DSP stacks are shown in Fig. 20.

We generate 30 GBaud QPSK or 16-QAM sequences with a length of $2^{12}$ in MATLAB. After up-sampling to 4 SPS and RRC filter with a roll-off factor of 0.01 , a weak digital carrier with different CSPR values is inserted in the edge of the signal spectrum $(-15.3 \mathrm{GHz})$ to obtain an initial phase at the receiver side. The digital pre-emphasis based on quadratic function [15] is used to pre-compensate the frequency response of AWG, EA, and IQ modulator. 


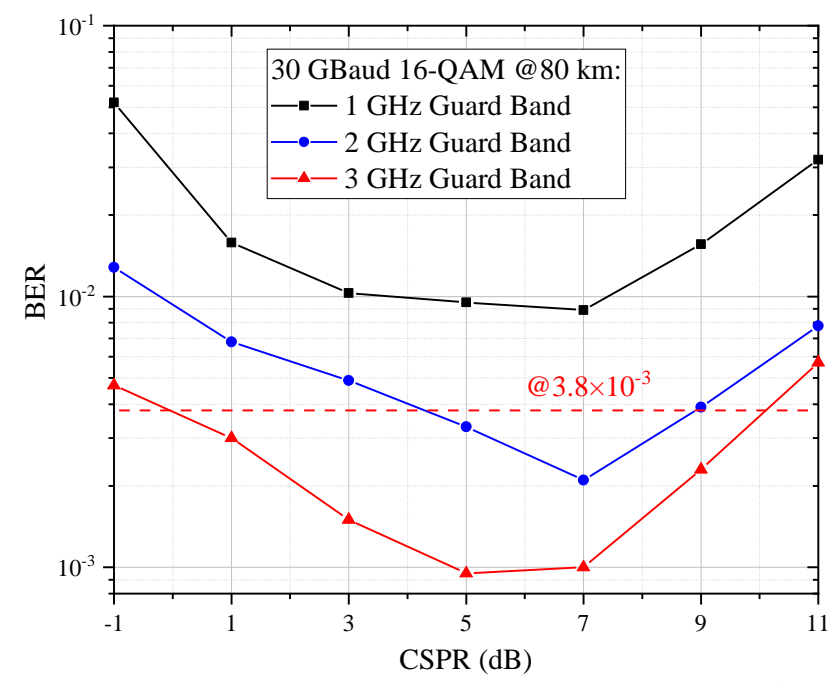

Fig. 18. Measured BERs versus CSPRs with $1 / 2 / 3 \mathrm{GHz}$ guard band after $80 \mathrm{~km}$ SMF transmission.

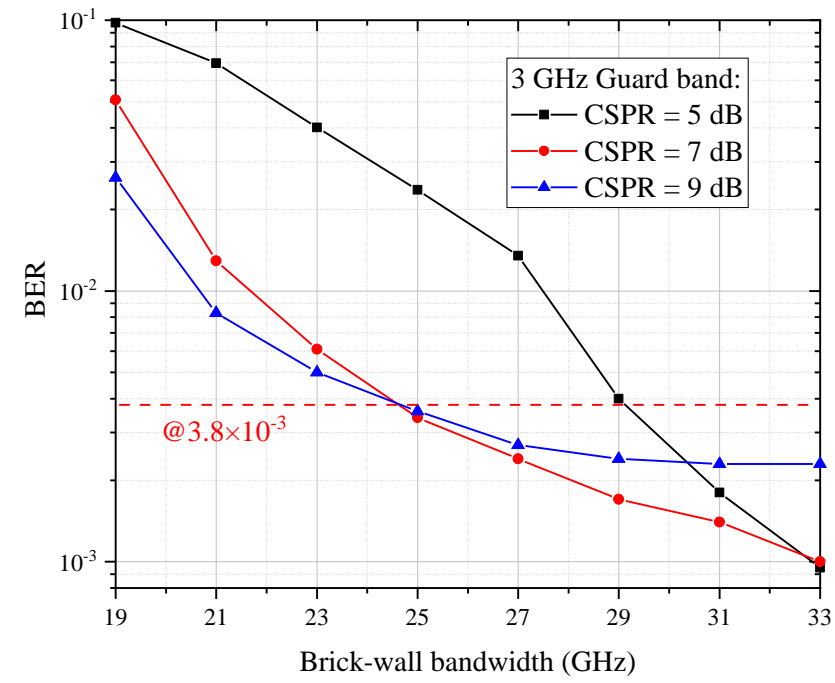

Fig. 19. Measured BERs versus the brick-wall electrical bandwidth at different CSPRs.

At the receiver DSP, the captured waveforms are resampled to $120 \mathrm{GSa} / \mathrm{s}$ for frame synchronization. After the two waveforms are synchronized and resampled to $90 \mathrm{GSa} / \mathrm{s}$ to avoid spectral overlap, the modified GS algorithm for ECA-PR (Table. II) is performed to retrieve the phase. The NOI is set to 15 to ensure complete convergence of the algorithm. Next, the signal is resampled to 2 SPS. After chromatic dispersion compensation and matched filtering, the sequences are fed into a feedforward equalizer (FFE) to eliminate ISI since the IQ modulator is always biased at the null point with ECA-PR scheme. The tap number is 31 , and the equalizer taps are updated by using the RLS algorithm based on 128 training symbols. After down-sampling and decision, the BER is calculated. The optical spectra with the CSPR from $-4 \mathrm{~dB}$ to 4 dB are depicted in Fig. 21.

The absolute phase error versus the NOI at different CSPRs is shown in Fig. 22. It shows the ECA-PR enables faster convergence than the CCA-PR. With the CSPR increasing from $-2 \mathrm{~dB}$ to $5 \mathrm{~dB}$, the convergence speed is accelerated and the required NOI to reach optimal performance is reduced. The

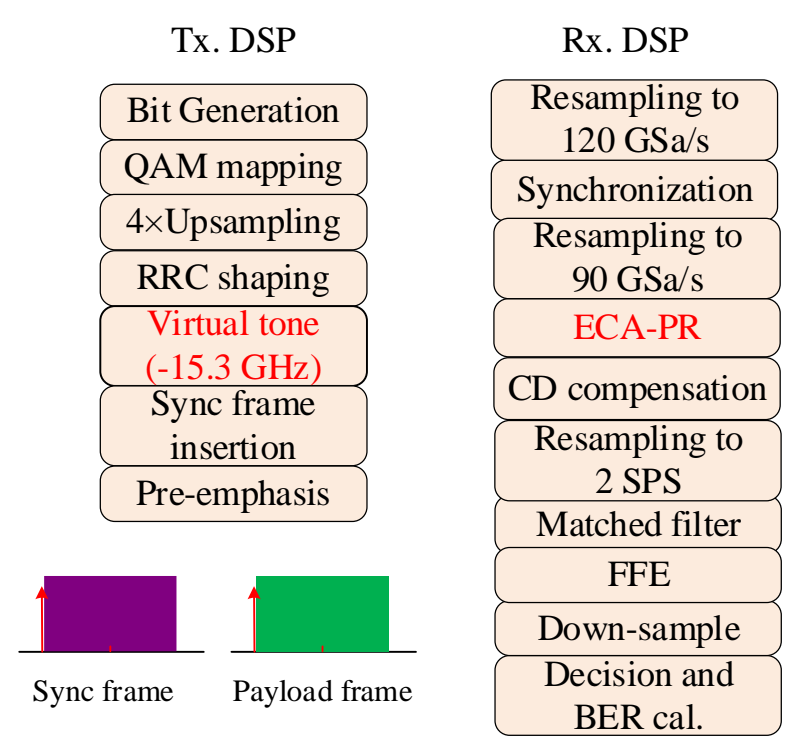

Fig. 20. DSP stacks for ECA-PR at the transmitter and receiver.

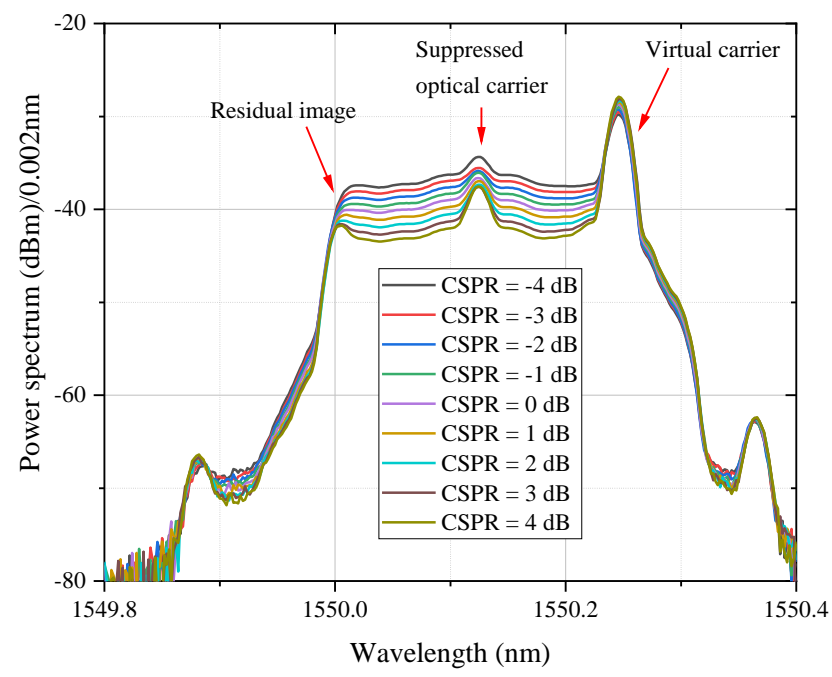

Fig. 21. Optical spectra of single carrier 30 GBaud 16-QAM signal with the CSPR from $-4 \mathrm{~dB}$ to $4 \mathrm{~dB}$.

absolute phase error from the PR algorithm is reduced by a higher CSPR, but the absolute phase error from the optical noise is enhanced.

The measured BERs as a function of CSPR with different applied dispersion values after $80 \mathrm{~km}$ transmission are shown in Fig. 23. For 30 GBaud QPSK signal, it indicates that the CSPR of $-1 \mathrm{~dB}$ is sufficient to reach a BER below the threshold of $7 \%$ HD-FEC with $-650 \mathrm{ps} / \mathrm{nm}$, which is consistent with the simulation. A larger applied dispersion value could further lower the required CSPR to $-2 \mathrm{~dB}$. For 30 GBaud 16-QAM signal, it requires the CSPR of $1 \mathrm{~dB}$ to reach a BER below $7 \%$ HD-FEC. A higher CSPR can help the ECA-PR to achieve optical field reconstruction but leads to a more significant OSNR penalty. The optimal CSPR is $3 \mathrm{~dB}$ for 16-QAM transmission with a BER of $6.4 \times 10^{-4}$. 


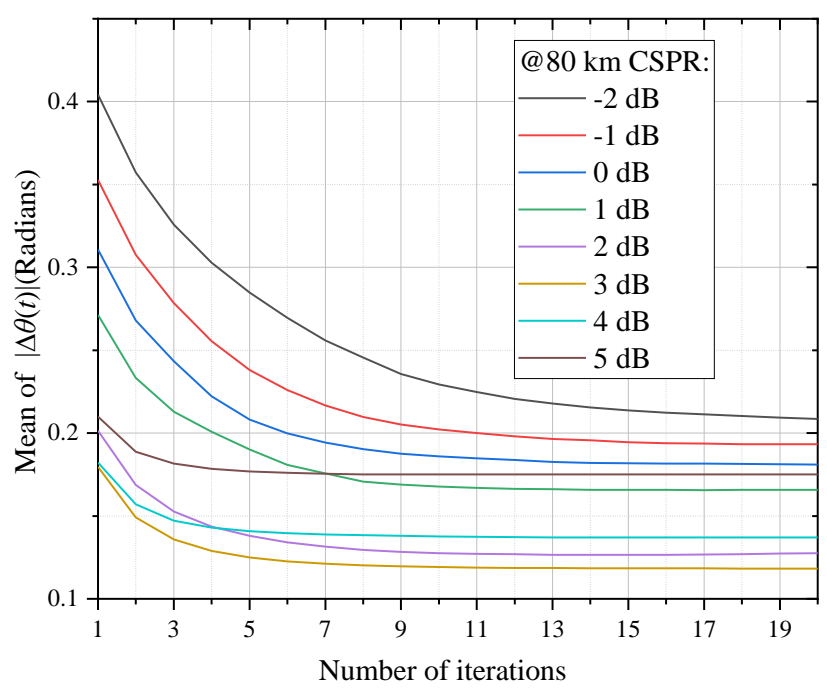

Fig. 22. Mean of $|\Delta \theta(t)|$ versus the number of iterations at different CSPRs after $80 \mathrm{~km}$ SMF transmission.

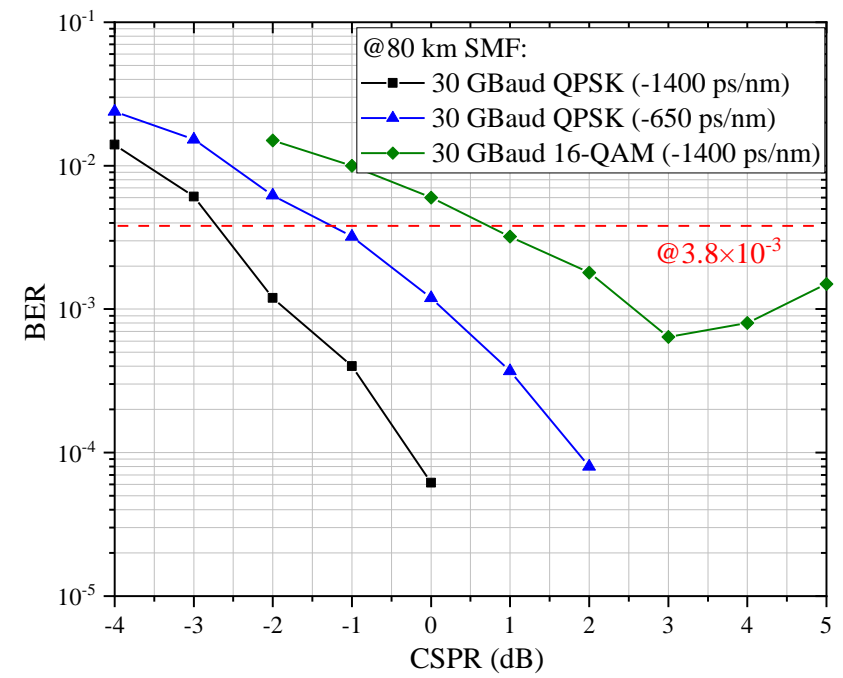

Fig. 23. Measured BERs as a function of CSPR with different applied dispersion value after $80 \mathrm{~km}$ transmission.

\section{DISCUSSION}

It is necessary for the long-haul and metro optical communication systems to take EDFAs as the optical amplifiers to extend the transmission reach. Therefore, the ASE noise is the dominant noise, and OSNR sensitivity is a critical parameter to measure receiver performance in the optical-amplified system. In this section, we analyze the OSNR sensitivity of some field recovery schemes for 30 GBaud 16-QAM transmission, including single-polarization (SP) intradyne coherent detection receiver (ICR), KKR, SVR, PR-PR, STD-PR, CCA-PR, and ECA-PR.

\section{A. OSNR sensitivity for 30 GBaud 16-QAM}

For the CCA-PR, the guard band for CAA-PR is $3 \mathrm{GHz}$ to match the previous experiment. The BERs of simulations and experiments versus OSNR are shown in Fig. 24. First, we analyze the results of numerical simulations.

Referring to [16], here a new definition can be used to account for the OSNR gap (dB) to the coherent system.

$$
\begin{aligned}
& \Delta=\Delta_{C S P R}+\Delta_{N F}+\Delta_{N}+\Delta_{P R} \\
& \Delta_{C S P R}=10 \times \log _{10}\left(1+10^{0.1 \times C S P R}\right)
\end{aligned}
$$

$\Delta_{C S P R}$ is the OSNR penalty from the carrier power. In this paper, $\Delta_{N F}$ is 0 for all the schemes assuming that an ideal optical rectangular filter is used before detection. In order to analyze the OSNR penalty of different FR methods in detail, we divide them into two categories: $\Delta_{N}$ and $\Delta_{P R} . \Delta_{N}$ is the penalty from the phase retrieval algorithm caused by ASE noise. It is $3 \mathrm{~dB}$ for the SVR induced by the noisy carrier on the orthogonal polarization and 0 for other schemes. $\Delta_{P R}$ is the OSNR penalty of the PR algorithm induced by the CSPR or the insufficient amount of dispersion. To simplify the comparison, we analyze the OSNR sensitivity of different FR schemes at a BER of $1 \times 10^{-3}$.

For the mainstream schemes such as KKR (6 dB CSPR) and SVR, there are $\sim 7.8 \mathrm{~dB}$ and $6 \mathrm{~dB}$ OSNR penalties, respectively, compared with the SP coherent detection. The detailed analysis can be found in Ref. [16].

As for the STD-PR, $\Delta_{D}$ is about $2 \mathrm{~dB}$ which results from the convergence error of the GS algorithm, which is partly induced by the insufficient ADV and the lack of diversity channels.

For the proposed PR schemes, the OSNR penalty can be divided into $\Delta_{C S P R}$ and $\Delta_{P R}$ as follows. For the CCA-PR with 1 $\mathrm{dB}$ CSPR and the ADV of $650 \mathrm{ps} / \mathrm{nm}$, the gap to SP ICR is 4.4 $\mathrm{dB}$, where $3.5 \mathrm{~dB}$ is from the carrier power and another $0.9 \mathrm{~dB}$ is $\Delta_{P R}$. Like KKR, a higher CSPR could help to reduce $\Delta_{P R}$. For the CCA-PR with $5 \mathrm{~dB}$ CSPR, the gap is about $6.5 \mathrm{~dB}$ where $6.2 \mathrm{~dB}$ is from the carrier and $0.3 \mathrm{~dB}$ is $\Delta_{P R}$ since the OSNR penalty of PR algorithm induced by the CSPR is neglectable at $5 \mathrm{~dB}$ CSPR. Comparing CCA-PR with $650 \mathrm{ps} / \mathrm{nm}$ and CCA-PR with $1400 \mathrm{ps} / \mathrm{nm}$ at $1 \mathrm{~dB}$ CSPR, the increase of ADV can reduce $\Delta_{P R}$ from $1.8 \mathrm{~dB}$ to $0.9 \mathrm{~dB}$. Therefore, for CCA-PR, the larger ADV could be used to reduce $\Delta_{P R}$ and the required CSPR. The reduction in CSPR leads to smaller $\Delta_{C S P R}$, which further improves the OSNR sensitivity. There is also a BER floor of $4 \times 10^{-4}$ for $1 \mathrm{~dB}$ CSPR and $650 \mathrm{ps} / \mathrm{nm}$ case, which can be eliminated by increasing the ADV or CSPR.

We then pay attention to the ECA-PR. For the ECA-PR, the CSPR is optimized under different OSNRs to match the experiment. The optimal CSPR is from $-2 \mathrm{~dB}$ to $0 \mathrm{~dB}$ for the ECA-PR with $1400 \mathrm{ps} / \mathrm{nm}$ dispersion, while it is from $-1 \mathrm{~dB}$ to $1 \mathrm{~dB}$ for the ECA-PR with $650 \mathrm{ps} / \mathrm{nm}$ dispersion. At the BER of $1 \times 10^{-3}$, the CSPR is $0 \mathrm{~dB}$ and $1 \mathrm{~dB}$ for $1400 \mathrm{ps} / \mathrm{nm}$ and 650 $\mathrm{ps} / \mathrm{nm}$ dispersion, respectively. This implies that a larger ADV can reduce the required CSPR and thus improve OSNR sensitivity. For the ECA-PR with $1400 \mathrm{ps} / \mathrm{nm}$, it has a $4 \mathrm{~dB}$ OSNR gap to SP coherent detection system at $1 \times 10^{-3}$, where there is a $3 \mathrm{~dB}$ intrinsic OSNR penalty that results from the weak carrier. Another $1 \mathrm{~dB}$ is $\Delta_{P R}$, resulting from the convergence error of the GS algorithm due to the insufficient amount of dispersion and CSPR. For the ECA-PR with 650 $\mathrm{ps} / \mathrm{nm}, \Delta_{C S P R}$ is about $3.5 \mathrm{~dB}$ at $1 \times 10^{-3}$, and thus the $\Delta_{P R}$ is $2 \mathrm{~dB}$. There is a BER floor of $1 \times 10^{-4}$ for $650 \mathrm{ps} / \mathrm{nm}$ case, which results from the insufficient ADV. Therefore, for the CCA-PR, phase recovery accuracy improves with larger dispersion, and 


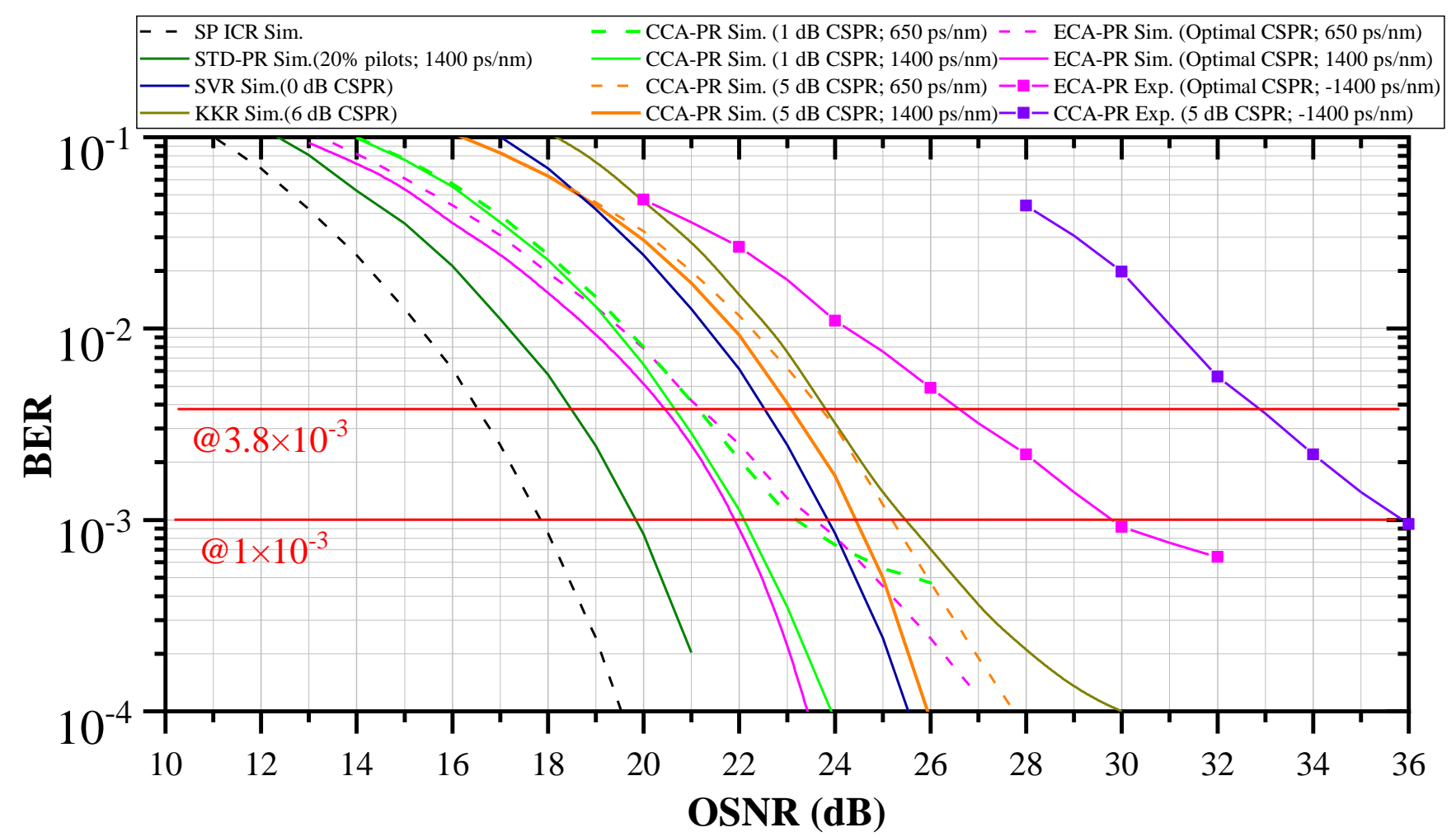

Fig. 24. OSNR sensitivity comparisons among SP ICR, KKR, SVR, STD-PR, ECA-PR and CCA-PR. The raw data rate is 120 Gb/s with 30 GBaud $16-Q A M$ modulation. Sim.: simulation; Exp.: experiment.

the larger ADV also could be used to reduce $\Delta_{P R}$ and the required CSPR.

The CCA-PR with $1 \mathrm{~dB}$ CSPR and $1400 \mathrm{ps} / \mathrm{nm}$ outperforms SVR by $1.6 \mathrm{~dB}$ since it is not affected by $\Delta_{N}$, and also shows 3.4 $\mathrm{dB}$ better than KKR due to the smaller CSPR. Note that the ESE could be improved by enhancing the carrier power in the CCA-PR, although it has the more significant OSNR penalty among the 3 PR schemes.

The ECA-PR with $1400 \mathrm{ps} / \mathrm{nm}\left(0 \mathrm{~dB}\right.$ CSPR at $\left.1 \times 10^{-3}\right)$ outperforms SVR by $2 \mathrm{~dB}$, and there is a $3.8 \mathrm{~dB}$ improvement over KKR with $6 \mathrm{~dB}$ CSPR. Note that although the OSNR sensitivity of ECA-PR is $2 \mathrm{~dB}$ lower than that of STD-PR, the net rate of ECA-PR is $20 \%$ higher due to the $20 \%$ pilot symbols.

\section{B. Implementation Penalty}

From the perspective of the field-deployed transceiver, the penalty between numerical simulation and experimental demonstration should be presented to illustrate the actual implementation difficulty of the proposed PR schemes. The measured BERs versus OSNR in the CCA-PR ( $5 \mathrm{~dB}$ CSPR and $-1400 \mathrm{ps} / \mathrm{nm}$ ) and the ECA-PR (optimal CSPR and $-1400 \mathrm{ps} / \mathrm{nm}$ ) are shown in Fig. 24. For the experimental results, we analyze the OSNR sensitivity of CCA-PR and ECA-PR at the 7\% HD-FEC BER threshold of $3.8 \times 10^{-3}$.

For the ECA-PR, the optimal CSPR is from $1 \mathrm{~dB}$ to $3 \mathrm{~dB}$ with OSNR increasing from $22 \mathrm{~dB}$ to $32 \mathrm{~dB}$. At the BER of $3.8 \times 10^{-3}$, the optimal CSPR is about $2 \mathrm{~dB}$. There is a $6 \mathrm{~dB}$ OSNR gap between the simulation and experiment, where 1.6 $\mathrm{dB}$ is from the carrier power and another $4.4 \mathrm{~dB}$ results from the imperfections of transmitter and receiver, including the bandwidth limitation and the effective number of bits of ADC.

For the CCA-PR, the OSNR gap is increased to about $10 \mathrm{~dB}$. The dominant reason for this is the electrical noise due to the lack of transimpedance amplifiers because the required CSPR is about $5 \mathrm{~dB}$ in the CCA-PR, which is higher than the ECA-PR. At the same received optical power, it requires a higher effective OSNR to enhance the tolerance to electrical noise. Another reason is the modulation nonlinearity because we control CSPR through the bias point of the IQ modulator, resulting in the modulation nonlinearity. Therefore, the ECA-PR with low CSPR is more preferred than CCA-PR based on the above analysis.

\section{Hardware Complexity}

We compare the hardware complexity of all the field recovery schemes mentioned in this paper. The transmitted optical signal bandwidth is $B$. The results are summarized in the Table. III. Among all schemes, only SP coherent detection needs a LO with narrow linewidth, and thus the receiver bandwidth should be slightly larger than $B / 2$ due to the frequency offset between lasers at the transmitter and receiver. The electrical bandwidth should also be larger than $B / 2$ for the schemes which need a guard band including CADD, ASCD, and CCA-PR. For the optical devices, the STD-PR requires the most hardware to complete carrier-less phase retrieval.

The SVR shows the highest ESE among all FR schemes because it realized FR in the Stokes-space, and the carrier is located at $0 \mathrm{GHz}$ on the orthogonal polarization. The STD-PR and ICR can support the most WDM channels due to the 
TABLE III

COMPARISONS AMONG $120 \mathrm{~Gb} / \mathrm{s}$ FIELD RECOVERY SCHEMES

\begin{tabular}{|c|c|c|c|c|c|c|c|c|c|c|}
\hline Scheme & Ref. & $\begin{array}{l}\mathrm{Tx} \\
\mathrm{BW} \\
\end{array}$ & LO & Key optical devices & $\mathrm{ADC}$ & PD & $\begin{array}{l}\mathrm{Rx} \\
\mathrm{BW} \\
\end{array}$ & $\begin{array}{l}\text { Pilot } \\
\text { Symbol }\end{array}$ & $\begin{array}{l}\text { Pilot } \\
\text { Tone }\end{array}$ & CSPR \\
\hline SP ICR & - & $B / 2$ & Yes & Optical hybrid & 2 & 2 BPDs & $>B / 2$ & Yes & $\mathrm{NO}$ & - \\
\hline KKR & {$[2]$} & $B / 2$ & No & - & 1 & SPD & $B$ & $\mathrm{NO}$ & Yes & 6 \\
\hline SVR & [3] & $B / 2$ & No & $\begin{array}{l}\text { Optical hybrid, PBS } \\
2 \text { Couplers }(1 \times 2)\end{array}$ & 3 & 3 BPDs & $B / 2$ & $\mathrm{NO}$ & Yes & 0 \\
\hline SVR & {$[16]$} & $B / 2$ & No & 2 Couplers $(1 \times 2,3 \times 3)$, PBS & 4 & 4 SPDs & $B / 2$ & $\mathrm{NO}$ & Yes & 0 \\
\hline STD-PR & {$[12]$} & $B / 2$ & No & $\begin{array}{l}2 \text { ODLs, Dispersive element } \\
2 \text { Couplers }(1 \times 4,3 \times 3) \\
\end{array}$ & 4 & 4 SPDs & $B$ & Yes & $\mathrm{NO}$ & - \\
\hline CADD & [4] & $>B / 2$ & No & $\begin{array}{l}\text { Optical hybrid, ODL } \\
2 \text { Couplers }(1 \times 2)\end{array}$ & 3 & 2 BPDs, SPD & $>B / 2$ & NO & Yes & $\sim 10$ \\
\hline ECA-PR & - & $B / 2$ & No & Dispersive element & & & $B$ & $\mathrm{NO}$ & Yes & -1 \\
\hline CCA-PR & - & $>B / 2$ & No & Coupler $(1 \times 2)$ & 2 & 2 SPDs & $>B / 2$ & $\mathrm{NO}$ & Yes & 1 \\
\hline $\mathrm{ASCD}$ & {$[5]$} & $>B / 2$ & No & & & & $>B / 2$ & $\mathrm{NO}$ & Yes & $\sim 10$ \\
\hline
\end{tabular}

Tx: transmitter; Rx: receiver; BW: bandwidth; LO: local oscillator; ODL: optical delay line; PBS: polarization beam splitter; ADC: analog-to-digital converter; PD: photodetector; SPD: single-ended photodetector; BPD: balanced photodetector; BW: bandwidth; CSPR: carrier-to-signal power ratio.

carrier-less characteristic. Only one PD and ADC are needed at the KKR. It shows the simplest hard-ware structure but the lowest spectral efficiency at the receiver. As for the carrier-assisted field recovery schemes, the CSPR is $1 \mathrm{~dB}$ and $-1 \mathrm{~dB}$ for CCA-PR and ECA-PR to realize PR, respectively. Therefore, the number of channels they can support is about half of the carrier-less schemes. The CCA-PR enables the flexibility between CSPR and ESE. Compared with CADD or ASCD, the CSPR of CCA-PR could be reduced significantly because the CADD and ASCD rely on SSBI construction and iterative cancellation algorithm [4-5]. These iterative reconstruction algorithms require a high CSPR in the first several iterations for convergence, while a low CSPR enables the CCA-PR to eliminate the phase ambiguity and realize field recovery. Each of these schemes has its own merits. It is a trade-off between hardware cost, CSPR, spectral efficiency, implementation difficulty, and computational complexity.

\section{CONCLUSION}

To improve the comprehensive performance of the phase retrieval schemes based on the GS algorithm, we propose two carrier-assisted phase retrieval schemes, CCA-PR and ECA-PR. These two schemes enable field recovery with fast convergence, low computational complexity, and low redundancy. We characterize the theoretical performance of CCA-PR and ECA-PR in the numerical simulations and investigate the impact of some critical parameters, including the applied dispersion value, CSPR, and electrical bandwidth. The CCA-PR offers fast convergence and flexibility between CSPR and electrical bandwidth requirement, while the ECA-PR enables faster convergence and lower CSPR than CCA-PR for phase retrieval. We experimentally demonstrate them for 30 GBaud 16-QAM transmission over $80 \mathrm{~km}$ with a BER below 7\% HD-FEC. Then we compare some mainstream field recovery schemes in terms of OSNR sensitivity, hardware cost, CSPR requirements, spectral efficiency, and implementation difficulty. Our work bridges the carrier-assisted self-coherent and carrier-less phase retrieval schemes with comprehensive analysis of the performance-complexity trade-off.

\section{REFERENCES}

[1] K. Kikuchi, "Fundamentals of Coherent Optical Fiber Communications," J. Lightw. Technol., vol.34, no.1, pp. 157-179, Jan. 2016.

[2] A. Mecozzi, C. Antonelli, and M. Shtaif, "Kramers-Kronig coherent receiver," Optica. vol.3, no. 11, pp. 1220-1227, Nov. 2016.

[3] D. Che, A. Li, X. Chen, Q. Hu, Y. Wang, and W. Shieh, "Stokes vector direct detection for linear complex optical channels," J. Lightw. Technol., vol. 33, no. 3, pp. 678-684, Feb. 2015.

[4] W. Shieh, C. Sun, and H. Ji, "Carrier-assisted differential detection," Light: Sci. Appl. vol. 9, no. 1, pp. 1-9, Feb. 2020.

[5] X. Li, M. O'Sullivan, Z. Xing, M. Alam, M. E. Mousa-Pasandi, and D. V. Plant, "Asymmetric self-coherent detection," Opt. Express. vol. 29, no. 16, pp. 25412-25427, Aug. 2021.

[6] R. W. Gerchberg, "A practical algorithm for the determination of phase from image and diffraction plane pictures," Optik, vol. 35, pp. 237-246, 1972.

[7] H. Chen, N. K. Fontaine, J. M. Gene, R. Ryf, D. T. Neilson and G. Raybon, "Dual Polarization Full-Field Signal Waveform Reconstruction Using Intensity Only Measurements for Coherent Communications, " $J$. Lightw. Technol., vol. 38, no. 9, pp. 2587-2597, May. 2020

[8] H. Chen, N. K. Fontaine, J. M. Gené, R. Ryf, D. T. Neilson and G. Raybon, "Full-field, carrier-less, polarization-diversity, direct detection receiver based on phase retrieval," Proc. Eur. Conf. Opt. Commun., Dublin, Ireland. 2019, Paper Tu1D.1.

[9] M. Xiang, P. Zhou, B. Ye, S. Fu, O. Xu, J. Li, D. Peng, Y. Wang, and Y. Qin, "Adaptive intensity transformation-based phase retrieval with high accuracy and fast convergence, " Opt. Lett. vol.46, no. 13, pp. 3215-3218, Jul. 2021.

[10] H. Huang, H. Chen, Y. Huang, N. K. Fontaine, R. Ryf, and Y. Song, "Carrier-less phase retrieval receiver leveraging digital upsampling," Opt. Lett. vol.45, no. 21, pp. 6070-6073, Nov. 2020.

[11] H. Chen, H. Huang, N. K. Fontaine, and R. Ryf, "Phase retrieval with fast convergence employing parallel alternative projections and phase reset for coherent communications, "Opt. Lett. vol.45, no.5, pp. 1188-1191, Mar. 2020.

[12] H. Chen, N. K. Fontaine, R. Essiambre, H. Huang, M. Mazur, R. Ryf, and D. T. Neilson, "Space-Time Diversity Phase Retrieval Receiver," Proc. OFC, San Francisco, CA, USA, 2021, Paper Th4D.3.

[13] Q. Wu, Y. Zhu, and W. Hu, "Weak carrier assisted phase retrieval receiver," Proc. OFC, San Diego, California, USA, 2022, Tu3B.6.

[14] X. Wu, A. S. Karar, K. Zhong, A. P. T. Lau, and C. Lu, "Experimental demonstration of pre-electronic dispersion compensation in IM/DD systems using an iterative algorithm, "Opt. Express. vol. 29, no. 16, pp. 24735-24749, Aug. 2021.

[15] D. Zou, Y. Chen, F. Li, Z. Li, Y. Sun, L. Ding, J. Li, X. Yi, L. Li, and Z. Li, "Comparison of Bit-Loading DMT and Pre-Equalized DFT-Spread DMT for 2-km Optical Interconnect System," J. Lightw. Technol., vol.37, no.10, pp. 2194-2200, May. 2019.

[16] D. Che, C. Sun, and W. Shieh, "Optical field recovery in stokes space," $J$. Lightw. Technol., vol. 37, no. 2, pp. 451-460, Jan. 2019. 